\title{
THEOREMS ON SEPARABILITY IN RIEMANNIAN $n$-SPACE
}

PARRY MOON AND DOMINA EBERLE SPENCER

Despite the importance of separation of variables as a means for solving partial differential equations, no comprehensive study of separability conditions seems to have been made. We limit investigation to the Helmholtz equation

$$
\nabla^{2} \phi+(k)^{2} \phi=0
$$

and the Laplace equation

$$
\nabla^{2} \phi=0
$$

and consider separability in an $n$-space with Riemannian metric $g_{i j}=0$, where $i \neq j$.

DEFINITION I. If the assumption

$$
\phi=\prod_{i=1}^{n} U^{i}\left(u^{i}\right)
$$

permits the separation of the partial differential equation into $n$ ordinary differential equations, the equation is said to be simply separable.

DEFINITION II. If the assumption

$$
\phi=\frac{1}{R\left(u^{1}, u^{2}, \cdots, u^{n}\right)} \prod_{i=1}^{n} U^{i}\left(u^{i}\right)
$$

permits the separation of the partial differential equation into $n$ ordinary differential equations, and if $R \neq$ const., the equation is said to be an $R$-separable.

\section{General case.}

THEOREM I. The necessary and sufficient conditions for simple separability of the Helmholtz equation, in Riemannian $n$-space with an orthogonal coordinate system and $\phi=\phi\left(u^{1}, u^{2}, \cdots, u^{n}\right)$, are

$$
\begin{aligned}
g_{i i} & =\frac{S}{M_{i 1}}, \\
\frac{g^{1 / 2}}{S} & =\prod_{i=1}^{n} f_{i}\left(u^{i}\right),
\end{aligned}
$$

Presented to the International Congress of Mathematicians, Cambridge, Mass., September 6, 1950; received by the editors November 28, 1951. 
where $S$ is the Stäckel determinant [1]

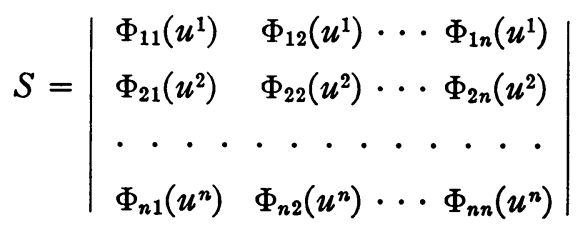

and $M_{i 1}$ is the cofactor of $\Phi_{i 1}$.

Proof. The Helmholtz equation may be written

$$
\frac{1}{g^{1 / 2}} \sum_{i=1}^{n} \frac{\partial}{\partial u^{i}}\left(\frac{g^{1 / 2}}{g_{i i}} \frac{\partial \phi}{\partial u^{i}}\right)+(k)^{2} \phi=0 .
$$

Evidently [2] a necessary condition for separation is

$$
\frac{g^{1 / 2}}{g_{i i}}=f_{i}\left(u^{i}\right) \cdot F_{i}\left(u^{1}, \cdots, u^{i-1}, u^{i+1}, \cdots, u^{n}\right) .
$$

Substitute Equation (7) in Equation (6) and differentiate with respect to the separation constants $\alpha_{1}=(k)^{2}, \alpha_{2}, \alpha_{3}, \cdots, \alpha_{n}$. Then we obtain a set of $n$ simultaneous algebraic equations,

$$
\sum_{i=1}^{n} f_{i} F_{i} \Phi_{i j}\left(u^{i}\right)=\delta_{j}^{1} g^{1 / 2}
$$

where

$$
\Phi_{i j}\left(u^{i}\right)=-\frac{1}{f_{i}\left(u^{i}\right)} \frac{\partial}{\partial \alpha_{j}}\left[\frac{1}{U^{i}} \frac{d}{d u^{i}}\left(f_{i} \frac{d U^{i}}{d u^{i}}\right)\right] .
$$

For $S \neq 0$, which is the only case of practical significance, the solution is

$$
f_{i} F_{i}=\frac{g^{1 / 2}}{S} M_{i 1}
$$

Combination of Equations (7) and (9) gives the conditions of Theorem I.

To show that these conditions are sufficient as well as necessary $[3 ; 4]$, we substitute Equation (5) into Equation (6) and find that separation is always effected, with the separated equations

$$
\frac{1}{f_{i}} \frac{d}{d u^{i}}\left(f_{i} \frac{d U^{i}}{d u^{i}}\right)+U^{i} \sum_{j=1}^{n} \alpha_{j} \Phi_{i j}=0, \quad i=1,2, \cdots, n .
$$

TheOREM II. The necessary and sufficient conditions for simple 
separability of the Laplace equation, in Riemannian $n$-space with an orthogonal coordinate system and $\phi=\phi\left(u^{1}, u^{2}, \cdots, u^{n}\right)$, are

$$
\frac{g_{i i}}{g_{i j}}=\frac{M_{i 1}}{M_{i 1}}, \quad \frac{g^{1 / 2}}{g_{i i}}=\left[\prod_{j=1}^{n} f_{j}\left(u^{j}\right)\right] M_{i 1} .
$$

Proof. Procedure is exactly as in the proof of Theorem I. The only difference is that the algebraic equations (8) are now homogeneous:

$$
\sum_{i=1}^{n} f_{i} F_{i} \Phi_{i j}\left(u^{i}\right)=0 .
$$

The solution therefore gives only ratios

$$
\frac{g_{i i}}{g_{j i}}=\frac{M_{j 1}}{M_{i 1}}
$$

and we have the lighter restrictions of Equation (11) as compared with those of Equation (5). As in the previous proof, the conditions are both necessary and sufficient, and the separated equations are again given by Equation (10).

THEOREM III. The necessary and sufficient conditions for $R$-separability of the Helmholtz equation, in Riemannian $n$-space with an orthogonal coordinate system and $\phi=\phi\left(u^{1}, u^{2}, \cdots, u^{n}\right)$, are

$$
\sum_{i=1}^{n} \frac{1}{f_{i} g_{i i}} \frac{\partial}{\partial u^{i}}\left(f_{i} \frac{\partial R}{\partial u^{i}}\right)+A R=0, \quad g_{i i}=\frac{S}{M_{i 1}},
$$

where $A$ is a constant and $R\left(u^{1}, u^{2}, \cdots, u^{n}\right)$ is determined by

$$
\frac{g^{1 / 2}}{S}=\left[\prod_{i=1}^{n} f_{i}\left(u^{i}\right)\right](R)^{2} \text {. }
$$

Proof. Substitution of Equation (4) into Equation (6) gives

$$
\sum_{i=1}^{n} \frac{1}{U^{i}} \frac{\partial}{\partial u^{i}}\left[\frac{g^{1 / 2}}{g_{i i}}\left(\frac{1}{R} \frac{d U^{i}}{d u^{i}}-\frac{U^{i}}{(R)^{2}} \frac{\partial R}{\partial u^{i}}\right)\right]+\frac{(k)^{2} g^{1 / 2}}{R}=0 .
$$

If terms in $\partial R / \partial u^{i}$ are to be separated from those in $d U^{i} / d u^{i}$, it is necessary that

$$
\frac{g^{1 / 2}}{g_{i i}}=(R)^{2} f_{i} F_{i}
$$

Substitution of Equation (15) into Equation (14) and multiplication by an arbitrary function $Q\left(u^{1}, u^{2}, \cdots, u^{n}\right)$ gives 


$$
(R)^{2} Q \sum_{i=1}^{n} \frac{F_{i}}{U^{i}} \frac{d}{d u^{i}}\left(f_{i} \frac{d U^{i}}{d u^{i}}\right)+\alpha_{1} g^{1 / 2}=0,
$$

where

$$
\alpha_{1}=(k)^{2} Q-\frac{R Q}{g^{1 / 2}} \sum_{i=1}^{n} F_{i} \frac{\partial}{\partial u^{i}}\left(f_{i} \frac{\partial R}{\partial u^{i}}\right) .
$$

Thus a necessary condition for separability is

$$
\sum_{i=1}^{n} \frac{1}{f_{i} g_{i i}} \frac{\partial}{\partial u^{i}}\left(f_{i} \frac{\partial R}{\partial u^{i}}\right)+\left(\frac{\alpha_{1}}{Q}-(k)^{2}\right) R=0 .
$$

Differentiation with respect to the separation constants leads to $n$ simultaneous equations

$$
\sum_{i=1}^{n} f_{i} F_{i} \Phi_{i j}\left(u^{i}\right)=\delta_{j}^{1} \frac{g^{1 / 2}}{(R)^{2} Q},
$$

the solution of which $(S \neq 0)$ is

$$
f_{i} F_{i}=\frac{g^{1 / 2}}{(R)^{2} Q} \frac{M_{i 1}}{S} .
$$

Comparison with Equation (15) shows that

$$
g_{i i}=\frac{S}{M_{i 1}} Q
$$

and

$$
\frac{g^{1 / 2}}{Q S}=\left(\prod_{i=1}^{n} f_{i}\left(u^{i}\right)\right)(R)^{2} .
$$

But it is easily shown that $Q=$ const., which may be taken as unity without loss of generality, and $\alpha_{1}=(k)^{2}+A$, where $A$ is an arbitrary contant. Thus Equations (16), (17), and (18) assume the form given in Theorem III.

If $k=0$, we obtain immediately:

THEOREM IV. The necessary and sufficient condition for $R$-separability of Laplace's equation, in Riemannian $n$-space with an orthogonal coordinate system and $\phi=\phi\left(u^{1}, u^{2}, \cdots, u^{n}\right)$, is

$$
\sum_{i=1}^{n} \frac{1}{f_{i} g_{i i}} \frac{\partial}{\partial u^{i}}\left(f_{i} \frac{\partial R}{\partial u^{i}}\right)+\alpha_{1} \frac{R}{Q}=0 ;
$$

where $R$ and $Q$ are defined by the relations 


$$
g_{i i}=\frac{S}{M_{i 1}} Q, \quad \frac{g^{1 / 2}}{Q S}=\left[\prod_{i=1}^{n} f_{i}\left(u^{i}\right)\right](R)^{2} .
$$

2. Degenerate cases. In most physical applications, $\phi$ is not a function of all $n$ coordinates but depends on a smaller number, say $m$. Without loss of generality, we number the coordinates $1,2, \cdots$, $m, \cdots, n$. Then the Helmholtz equation becomes

$$
\frac{1}{g^{1 / 2}} \sum_{1}^{m} \frac{\partial}{\partial u^{i}}\left(\frac{g^{1 / 2}}{g_{i i}} \frac{\partial \phi}{\partial u^{i}}\right)+(k)^{2} \phi=0
$$

and the solution for simple separability is

$$
\phi=U^{1} \cdot U^{2} \cdots U^{m} ;
$$

but $g_{i i}$ and $g^{1 / 2}$ may still contain all $n$ variables, and

$$
\frac{g^{1 / 2}}{g_{i i}}=f_{i}\left(u^{i}\right) \cdot F_{i}\left(u^{1}, u^{2}, \cdots, u^{i-1}, u^{i+1}, \cdots, u^{n}\right) .
$$

Separability conditions are considerably lightened:

THEOREM V. The necessary and sufficient conditions for simple separability of the Helmholtz equation, in Riemannian $n$-space with an orthogonal coordinate system and $\phi=\phi\left(u^{1}, u^{2}, \cdots, u^{m}\right)$, are

$$
\begin{array}{rlr}
g_{i i} & =\frac{S}{M_{i 1}}, & i=1,2, \cdots, m, \\
\frac{g^{1 / 2}}{S} & =\left[\prod_{i=1}^{m} f_{i}\left(u^{i}\right)\right] \cdot \mathcal{F}\left(u^{m+1}, \cdots, u^{n}\right), &
\end{array}
$$

where

$$
S=\left|\begin{array}{ccc}
\Phi_{11} & \cdots & \Phi_{1 m} \\
\cdot & \cdots & \cdot \\
\Phi_{m 1} & \cdots & \Phi_{m m}
\end{array}\right|
$$

Proof. Though the space remains $n$-dimensional, there are only $m$ separation constants. Thus Equation (8) becomes

$$
\sum_{i=1}^{m} f_{i} F_{i} \Phi_{i j}\left(u^{i}\right)=\delta_{j}^{1} g^{1 / 2}
$$

and the Stäckel matrix contains only $m^{2}$ terms. Procedure as in Theorem I results in Theorem V. Similarly, we have for the Laplace equation: 
TheOREM VI. The necessary and sufficient conditions for simple separability of the Laplace equation, in Riemannian $n$-space with an orthogonal coordinate system and $\phi=\phi\left(u^{1}, u^{2}, \cdots, u^{m}\right)$, are

$$
\begin{aligned}
\frac{g_{i i}}{g_{j j}} & =\frac{M_{i 1}}{M_{i 1}}, & i, j=1,2, \cdots, m, \\
\frac{g^{1 / 2}}{g_{i i}} & =\left[\prod_{j=1}^{m} f_{j}\left(u^{j}\right)\right] \cdot \mathcal{F}\left(u^{m+1}, \cdots, u^{n}\right), &
\end{aligned}
$$

where $M_{i 1}$ and $M_{j 1}$ are cofactors in the $m \times m$ Stäckel matrix of Theorem V.

As a special case, we have $m=1$. The question of separation does not arise, but a one-dimensional solution is possible only under certain conditions. Evidently the requirements are obtained from Equations (7) and (6):

TheOREM VII. The necessary and sufficient conditions that the Helmholtz equation have a one-dimensional solution $\phi=\phi\left(u^{j}\right)$, in Riemannian $n$-space with an orthogonal coordinate system, are

(24) $\frac{g^{1 / 2}}{g_{j i}}=f_{j}\left(u^{j}\right) \cdot F_{j}\left(u^{1}, \cdots, u^{j-1}, u^{j+1}, \cdots, u^{n}\right), \quad g_{i j}=g_{i j}\left(u^{j}\right)$.

TheOREM VIII. The necessary and sufficient condition that the Laplace equation have a one-dimensional solution $\phi=\phi\left(u^{i}\right)$, in Riemannian $n$-space with an orthogonal coordinate system, is that (21) holds.

3. Euclidean 3-space. Separability conditions in euclidean 3-space are obtained directly from the theorems of $\$ \$ 1$ and 2 . In a number of special cases, however, the requirements are so greatly simplified that they should be mentioned. In any orthogonal cylindrical coordinate system in 3-space, with $g_{11}=g_{22}$, the Stäckel matrix may be written

$$
[S]=\left[\begin{array}{lll}
0 & \Phi_{12} & \Phi_{13} \\
0 & \Phi_{22} & \Phi_{23} \\
1 & 0 & 1
\end{array}\right]
$$

The corresponding matrix for any orthogonal rotational system in 3 -space, written with $g_{11}=g_{22}$, is

$$
[S]=\left[\begin{array}{lll}
\Phi_{11} & \Phi_{12} & \Phi_{13} \\
\Phi_{21} & \Phi_{22} & \Phi_{23} \\
0 & 0 & 1
\end{array}\right]
$$


The following theorems are obvious consequences of $\$ \$ 1$ and 2:

THEOREM IX. The Helmholtz and Laplace equations are never $R$ separable in any orthogonal cylindrical coordinate system in euclidean 3-space.

THEOREM X. The necessary and sufficient condition for simple separability of the Helmholtz or the Laplace equation, in euclidean 3-space with an orthogonal cylindrical coordinate system, $g_{11}=g_{22}$, and $\phi$ $=\phi\left(u^{1}, u^{2}, u^{3}\right)$, is that the metric coefficient be a separable sum:

$$
g_{11}=-\left[\Phi_{13}\left(u^{1}\right)+\Phi_{23}\left(u^{2}\right)\right] \text {. }
$$

THEOREM XI. The necessary and sufficient conditions for simple separability of the Helmholtz equation, in euclidean 3-space with an orthogonal rotational coordinate system, $g_{11}=g_{22}$, and $\phi=\phi\left(u^{1}, u^{2}, u^{3}\right)$, are

$$
\begin{gathered}
g_{11}=\Phi_{11}\left(u^{1}\right)+\Phi_{21}\left(u^{2}\right), \quad \frac{g_{11}}{g_{33}}=-\left[\Phi_{13}\left(u^{1}\right)+\Phi_{23}\left(u^{2}\right)\right], \\
g_{33}=\left[f_{1}\left(u^{1}\right) \cdot f_{2}\left(u^{2}\right)\right]^{2} .
\end{gathered}
$$

ThEOREM XII. The necessary and sufficient conditions for simple separability of the Laplace equation, in euclidean. 3-space with an orthogonal rotational coordinate system, $g_{11}=g_{22}$, and $\phi=\phi\left(u^{1}, u^{2}, u^{3}\right)$, are

$$
\frac{g_{11}}{g_{33}}=-\left[\Phi_{13}\left(u^{1}\right)+\Phi_{23}\left(u^{2}\right)\right], \quad g_{33}=\left[f_{1}\left(u^{1}\right) \cdot f_{2}\left(u^{2}\right)\right]^{2} .
$$

THEOREM XIII. Simple separability of the Laplace equation occurs in every orthogonal cylindrical coordinate system in euclidean 3-space with $\phi=\phi\left(u^{1}, u^{2}\right)$.

THEOREM XIV. The necessary and sufficient condition for simple separability of the Helmholtz equation, in euclidean 3-space with orthogonal cylindrical coordinates, $g_{11}=g_{22}$, and $\phi=\phi\left(u^{1}, u^{2}\right)$, is

$$
g_{11}=\Phi_{11}\left(u^{1}\right)+\Phi_{21}\left(u^{2}\right) \text {. }
$$

THEOREM XV. The necessary and sufficient conditions for simple separability of the Helmholtz equation, in euclidean 3-space with an orthogonal rotational coordinate system, $g_{11}=g_{22}$, and $\phi=\phi\left(u^{1}, u^{2}\right)$, are

$$
g_{11}=\Phi_{11}\left(u^{1}\right)+\Phi_{21}\left(u^{2}\right), \quad g_{33}=\left[f_{1}\left(u^{1}\right) \cdot f_{2}\left(u^{2}\right)\right]^{2} .
$$

TheOREM XVI. The necessary and sufficient condition for simple separability of the Laplace equation, in euclidean 3-space with an 
orthogonal rotational coordinate system, $g_{11}=g_{22}$, and $\phi=\phi\left(u^{1}, u^{2}\right)$, is

$$
g_{33}=\left[f_{1}\left(u^{1}\right) \cdot f_{2}\left(u^{2}\right)\right]^{2} .
$$

TheOREM XVII. The necessary and sufficient conditions for $R$-separability of the Helmholtz equation, in euclidean 3-space with an orthogonal rotational coordinate system, $g_{11}=g_{22}$ and $\phi=\phi\left(u^{1}, u^{2}\right)$, are

$$
g_{11}=\Phi_{11}\left(u^{1}\right)+\Phi_{21}\left(u^{2}\right), \quad \frac{g_{11}}{g_{33}}=\Omega_{1}\left(u^{1}\right)+\Omega_{2}\left(u^{2}\right),
$$

where $\Omega_{1}$ and $\Omega_{2}$ are arbitrary functions.

TheOREM XVIII. The necessary and sufficient condition for $R$ separability of the Laplace equation, in euclidean 3-space with an orthogonal rotational coordinate system, $g_{11}=g_{22}$, and $\phi=\phi\left(u^{1}, u^{2}\right)$, is

$$
\frac{g_{11}}{g_{33}}=\Omega_{1}\left(u^{1}\right)+\Omega_{2}\left(u^{2}\right) \text {. }
$$

\section{BIBLIOGRAPHY}

1. Paul Stäckel, Über die Integration der Hamilton-Jacobischen Differentialgleichung mittels Separation der Variabelen, Habilitationsschrift, Halle, 1891.

- Sur une classe de problèmes de dynamique, C.R. Acad. Sci. Paris vol. 116 (1893) p. 485.

, Über die Integration der Hamiltonschen Differentialgleichung mittels Separation der Variabelen, Math. Ann. vol. 49 (1897) p. 145.

2. H. P. Robertson, Bemerkung über separierbare Systeme in der Wellenmechanik, Math. Ann. vol. 98 (1927) p. 749.

3. L. P. Eisenhart, Separable systems of Stäckel, Ann. of Math. vol. 35 (1934) p. 284.

p. 57.

, Stäckel systems in conformal euclidean space, Ann. of Math. vol. 36 (1935)

4. P. M. Morse and H. Feshbach, Methods of theoretical physics, Cambridge, Technology Press, 1946.

Massachusetts Institute of TechNology and

UNIVERSITY OF CONNECTICUT 